\title{
Uso de terapia antirretroviral por pacientes com HIV/Aids: associações com variáveis demográficas e clínicas
}

\author{
Use of antiretroviral therapy by HIV/Aids patients: associations with demographic and \\ clinical variables
}

Uso de terapia antirretroviral por pacientes com VIH/Sida: asociaciones con variables demográficas y clínicas

Kamille Karolinne Sagrilo ${ }^{1}$, Amanda Salles Margatho ${ }^{1}$, Rejane Kiyomi Furuya ${ }^{2}$, Danielle Cortêz da Silva ${ }^{1}$, Ana Beatriz Floriano de Souza ${ }^{1}$, Rafaela Salviato Cavéquia ${ }^{1}$, Rafaela Marioto Montanha ${ }^{1 *}$, Jéssica Maia Storer ${ }^{1}$, Gilselena Kerbauy ${ }^{1}$, Flávia Meneguetti Pieri¹.

\section{RESUMO}

Objetivo: Avaliar o uso de terapia antirretroviral em pacientes com HIV/Aids e associar esses níveis com as variáveis demográficas e clínicas. Assim como avaliar os principais motivos de internação de pacientes com HIV/Aids. Métodos: Estudo epidemiológico, observacional, retrospectivo, realizado em hospital público de ensino e referência para diagnóstico e tratamento de infecções sexualmente transmissíveis. A amostra foi de 154 prontuários de pessoas vivendo com HIV/Aids, internados em 2015. Resultados: A maioria não fazia uso de terapia antirretroviral $(n=107 ; 68,6 \%)$. O não uso de antirretrovirais foi mais frequente em indivíduos jovens $(p=0,002)$, com CD4+ menor que $200(p=0,002)$, carga viral maior que $10.000(p<0,0001)$ e tempo de diagnóstico menor que um ano $(p=0,002)$. Os principais motivos de internação dos participantes do estudo foram infecções oportunistas, com destaque para tuberculose, toxoplasmose cerebral, monilíase e pneumonia. Conclusão: Deve-se implementar medidas para aumentar o uso de terapia antirretroviral, especialmente em indivíduos jovens e naqueles com menor tempo de diagnóstico, sendo necessário o fortalecimento do vínculo desses indivíduos com a equipe e serviço de saúde.

Palavras-chave: HIV, Síndrome de Imunodeficiência Adquirida, Adesão à medicação, Morte, Infecção oportunista.

\begin{abstract}
Objective: Assess the use of antiretroviral therapy in HIV/Aids patients and associate these levels with demographic and clinical variables; as well as to assess the main reasons for hospitalization of HIV/Aids patients. Methods: Epidemiological, observational, retrospective study, developed at a public teaching hospital that is a reference for the diagnosis and treatment of sexually transmissible diseases. The sample consisted of 154 patient files of people living with HIV/Aids, hospitalized in 2015. Results: The majority did not use antiretroviral therapy ( $\mathrm{N}=107 ; 68.6 \%)$. The non-use of antiretroviral drugs was more frequent in young people $(p=0.002)$, with CD4+ inferior to $200(p=0.002)$, viral load superior to $10,000(p<0.0001)$ and time of diagnosis under one year $(p=0.002)$. The main reasons for the study participants' hospitalization were opportunistic infections, particularly tuberculosis, neurotoxoplasmosis, moniliasis and pneumonia. Conclusion: Measures are needed to increase the use of antiretroviral therapy, especially in young people and individuals diagnosed more recently. These people's bond with the health team and service needs strengthening.
\end{abstract}

Keywords: HIV, Acquired Immunodeficiency Syndrome, Medication adherence, Death, Opportunistic infection.

\footnotetext{
${ }^{1}$ Universidade Estadual de Londrina (UEL), Londrina - PR. *E-mail: rafaela.montanha@hotmail.com

2 Instituto Federal do Paraná (IFPR), Londrina - PR.
} 
RESUMEN

Objetivo: Evaluar el uso de terapia antirretroviral en pacientes con $\mathrm{VIH} /$ Sida y asociar esos niveles con las variables demográficas y clínicas, así como evaluar los principales motivos de hospitalización de pacientes con VIH/Sida. Métodos: Estudio epidemiológico, observacional, retrospectivo, desarrollado en hospital público de enseñanza y referencia para diagnóstico y tratamiento de infecciones sexualmente transmisible. La muestra fue formada por 154 archivos de personas con VIH/Sida, internadas en 2015. Resultados: La mayoría no usaba terapia antirretroviral $(n=107 ; 68,6 \%)$. El no uso de antiretrovirales fue más frecuente en individuos jóvenes ( $p=0,002)$, con CD4+ inferior a $200(p=0,002)$, carga viral mayor que $10.000(p<0,0001)$ y tiempo de diagnóstico inferior a un año $(p=0,002)$. Los principales motivos de hospitalización de los participantes fueron infecciones oportunistas, destacándose la tuberculosis, toxoplasmosis cerebral, moniliasis y neumonía. Conclusión: Se deben implementar medidas para aumentar el uso de terapia antirretroviral, especialmente en individuos jóvenes y en aquellos con menor tiempo de diagnóstico, siendo necesario fortalecer el vínculo de esos individuos con el equipo y el servicio de salud.

Palabras-clave: VHI, Síndrome de Inmunodeficiencia Adquirida, Adhesión a la medicación, Muerte, Infección oportunista.

\section{INTRODUÇÃO}

A infecção pelo vírus da imunodeficiência humana e a síndrome da imunodeficiência adquirida (HIV/Aids) é uma epidemia para a saúde pública. Segundo dados da Joint United Nations Programme on HIV/Aids (UNAIDS), mundialmente, até 2018, as estimativas eram de 37,9 milhões de pessoas vivendo com HIV (PVHIV), sendo 1,7 milhão de novas infecções (UNAIDS, 2020).

Apesar de números expressivos, a prevalência do HIV/Aids no Brasil apresentou queda nos últimos anos, de 20,7 para 18,5 casos a cada 100.000 habitantes nos anos de 2007 e 2016 respectivamente. Houve aumento expressivo no número de PVHIV em tratamento, passando de 183.997 em 2008 para 494.182 em 2016, além da redução do diagnóstico tardio, observado pela contagem de linfócitos TCD4+ inferior a 350 células $/ \mathrm{mm}^{3}$ no momento do diagnóstico, que reduziu de $28,6 \%$ em 2010 para $22,8 \%$ em 2016. Ressalta-se, que o Brasil foi o primeiro país a implementar a distribuição universal de medicamentos para o tratamento do HIV/Aids, sendo considerado uma das referências a nível mundial (BRASIL, 2019).

Com objetivo de erradicar a epidemia, a UNAIDS em parceria com diversos países inclusive o Brasil, assumiu o compromisso dos Objetivos de Desenvolvimento do Milênio, as chamadas metas 90-90-90 que referem à: 90\% de PVHIV devem ser diagnosticadas, 90\% das PVHIV diagnosticadas devem estar em tratamento e $90 \%$ das PVHIV em tratamento apresentando supressão viral. Quando esta meta tríplice for alcançada, pelo menos 73\% de todas as PVHIV no mundo terão supressão viral (UNAIDS, 2015).

Portanto, para atingir a meta proposta, é de extrema necessidade que as PVHIV tenham plena adesão a Terapia Antirretroviral (TARV), a fim de promover diminuição do potencial de transmissão da infecção pelo vírus de forma coletiva e redução dos óbitos (UNAIDS, 2015). Estudos demonstram que a não adesão ou adesão insuficiente ocorre tanto em países subdesenvolvidos como desenvolvidos, e a taxa média de não adesão é de $50 \%$. Esse valor se mostra preocupante, devido à alta e constante taxa de replicação e mutação do HIV, sendo necessário, pelo menos 95\% de adesão, para manter a Carga Viral (CV) indetectável (SILVA HFN, et al., 2019; RAVANHOL GM, et al., 2019).

Os fatores que podem interferir na adesão são variáveis relacionadas ao indivíduo (idade, gênero, escolaridade), variáveis relacionadas à TARV (esquema de medicação, efeitos adversos, esquecimento); aquelas relacionadas às características clínicas do HIV/Aids (tempo de diagnóstico e condições gerais de saúde); e as relacionadas ao acesso ao tratamento e serviços de assistência (presença de equipe multidisciplinar, visitas domiciliares, intervalo de tempo entre consultas, distância do local de consulta, existência de farmácias especializadas) (CARVALHO PP, et al., 2019).

Considerando a relevância da adesão à TARV para eficácia do tratamento, melhoria da qualidade de vida, redução das morbidades e aumento da sobrevida das PVHIV destaca-se a importância de estudos que visem caracterizar os fatores relacionados ao uso da TARV (SOUZA HC, et al., 2019). 
Ademais, o Ministério da Saúde (2018), ressalta o impacto a partir da ampliação do acesso à testagem e redução do tempo entre o diagnóstico e o início do tratamento, corroborando para a redução da mortalidade. Destaca-se, ainda, a importância de circular informações apropriadas sobre o tema HIV/Aids para que haja a construção e divulgação de atitudes e saberes para adoção de medidas preventivas, assim como para a desconstrução de tabus e estereótipos (MORAES TM, et al., 2019).

Além disso, estudos sobre o uso da TARV fornecem, aos gestores públicos, fontes de informações confiáveis para planejar investimentos em políticas públicas focadas na adesão ao tratamento (CARVALHO PP, et al., 2019), contribuindo para implantação de estratégias que otimizem e qualifiquem a produção do cuidado em saúde.

Este estudo objetivou avaliar o uso da TARV em pacientes com HIV/Aids por meio de características demográficas e clínicas, assim como verificar os principais motivos de internação de PVHIV/Aids.

\section{MÉTODOS}

Trata-se de um estudo observacional, retrospectivo e descritivo, mediante a consulta de prontuário. O método de estudo foi norteado pelo Strengthening Reporting of Observational Studies in Epidemiology (STROBE) Statement (MALTA M, et al., 2010). O estudo foi realizado em uma Unidade de Internação de Moléstias Infecciosas de um Hospital Universitário, o qual é referência para diagnóstico e tratamento de Infecções Sexualmente Transmissíveis.

Uma amostra consecutiva e não probabilística foi constituída de PVHIV, internadas no ano de 2015, sendo avaliados 420 prontuários. Os critérios de inclusão foram todos os prontuários de PVHIV com a primeira internação no ano de 2015, adultos, de ambos os sexos, totalizando 160 prontuários analisados. Desses, quatro não continha informação sobre o uso de TARV, e foram excluídos. Dessa forma, a amostra constituiu de 156 prontuários de PVHIV/Aids. Recorreu-se, também, ao banco de dados do Serviço de Arquivamento Médico e Estatístico (SAME), disponível na instituição para conferência do histórico de internações da amostra. Para confirmar o diagnóstico de HIV/Aids, foi verificado se os sujeitos haviam sido notificados no Sistema de Informação de Agravos de Notificação disponibilizado pela Vigilância Epidemiológica do município.

A coleta de dados foi realizada no período de agosto de 2016 à abril de 2017. Foram coletados os seguintes dados demográficos e clínicos, por meio dos prontuários: sexo, idade, orientação sexual, cor autorreferida, situação conjugal, escolaridade, procedência, uso de drogas, uso de TARV, tempo de diagnóstico, número de internações e motivo de internações. Os dados laboratoriais de contagem de Linfócitos TCD4+/CD8 e CV foram consultados no Módulo de Visualização de Exames da Alfa, disponibilizado na instituição. Para avaliar o número e o motivo de internações, foi realizada análise dos dados dos prontuários desde a primeira internação, ocorrida em 2015, no hospital em que o estudo foi realizado.

O uso de TARV foi avaliado por meio do autorrelato do paciente descrito no prontuário, por meio das evoluções médicas e de enfermagem. A prescrição de TARV nos prontuários foi verificado nas prescrições médicas e suas respectivas evoluções, sendo que a TARV era prescrita somente aos pacientes que já estavam fazendo uso do medicamento. As informações referentes ao uso de TARV também foram confirmadas nos serviços de contra referência do município, que realizam o acompanhamento de PVHIV. Assim, foram considerados como pacientes que faziam uso de TARV aqueles com autorrelato de uso, associado à prescrição médica e confirmação pelo serviço de contra referência.

Os dados foram transportados para uma planilha Excel for Windows 2003 e analisados no software Statistical Package for the Social Science (SPSS) versão 20.0 de forma descritiva. Para analisar as associações entre o uso de TARV com variáveis demográficas e clínicas utilizou-se o teste Qui-quadrado, com nível de significância de $5 \%$.

O estudo está vinculado a um projeto maior intitulado "Núcleo de Vigilância Epidemiológica: agravos transmissíveis notificados em Londrina, Paraná", aprovado pelo Comitê de Ética em Pesquisa em Seres Humanos da Universidade Estadual de Londrina, obtendo o parecer 110046/2015 e CAAE: 50559815.6.0000.5231. Esta pesquisa foi realizada seguindo os princípios éticos contidos na Resolução n. 466/2012 do Conselho Nacional de Saúde. 


\section{RESULTADOS}

Dos 156 participantes do estudo, a maioria era do sexo masculino, com 40 anos ou mais. Houve predomínio de indivíduos heterossexuais, de cor autorreferida branca ou amarela, com estudo até o ensino fundamental e sem companheiro. A maioria não fazia uso de TARV. Houve associação estatisticamente significante entre idade e uso de TARV $(p=0,002)$, sendo mais frequente o não uso em indivíduos mais jovens (Tabela 1).

Tabela 1 - Análise descritiva das características sociodemográficas das 156 pessoas vivendo com HIV (PVHIV), conforme uso de terapia antirretroviral (TARV) e valores de probabilidade ( $p$ ) associados ao teste de Qui-quadrado, 2017.

\begin{tabular}{|c|c|c|c|c|c|}
\hline \multirow{3}{*}{ Variáveis sociodemográficas } & \multicolumn{4}{|c|}{ Uso de TARV } & \multirow{3}{*}{$\begin{array}{l}\text { Valor } \\
\text { de } p\end{array}$} \\
\hline & \multicolumn{2}{|c|}{$\underset{(n=49)}{\operatorname{Sim}}$} & \multicolumn{2}{|c|}{$\begin{array}{c}\text { Não } \\
(n=107)\end{array}$} & \\
\hline & $\mathbf{n}$ & $\%$ & $\mathbf{n}$ & $\%$ & \\
\hline \multicolumn{6}{|l|}{ Sexo $(n=156)$} \\
\hline Masculino & 29 & 30,5 & 66 & 69,5 & \multirow{2}{*}{0,77} \\
\hline Feminino & 20 & 32,8 & 41 & 67,2 & \\
\hline \multicolumn{6}{|l|}{ Idade $(n=153)$} \\
\hline Até 39 anos & 12 & 18,2 & 54 & 81,8 & \multirow{2}{*}{0,002} \\
\hline 40 ou mais & 36 & 41,4 & 51 & 58,6 & \\
\hline \multicolumn{6}{|l|}{ Orientação sexual (n=146) } \\
\hline Heterossexual & 37 & 31,6 & 80 & 68,4 & \multirow{2}{*}{0,77} \\
\hline Homossexual, bissexual, transexual & 10 & 34,5 & 19 & 65,5 & \\
\hline \multicolumn{6}{|l|}{ Cor autorreferida(n=155) } \\
\hline Branca ou amarela & 28 & 34,6 & 53 & 65,4 & \multirow{2}{*}{0,41} \\
\hline Preta, parda ou indígena & 21 & 28,4 & 53 & 71,6 & \\
\hline \multicolumn{6}{|l|}{ Situação conjugal ( $n=153)$} \\
\hline Solteiro, divorciado ou viúvo & 32 & 30,5 & 73 & 69,5 & \multirow{2}{*}{0,54} \\
\hline Casado ou amasiado & 17 & 35,4 & 31 & 64,6 & \\
\hline \multicolumn{6}{|l|}{ Escolaridade $(n=129)$} \\
\hline Pouco estudo ou ensino fundamental & 25 & 28,7 & 62 & 71,3 & \multirow{2}{*}{0,59} \\
\hline Ensino médio ou superior & 14 & 33,3 & 28 & 66,7 & \\
\hline \multicolumn{6}{|l|}{ Procedência $(n=149)$} \\
\hline Atenção primária à saúde & 10 & 25,0 & 30 & 75,0 & \multirow{4}{*}{0,60} \\
\hline Hospitais & 9 & 29,0 & 22 & 71,0 & \\
\hline Serviço de assistência especializada & 7 & 41,2 & 10 & 58,8 & \\
\hline $\begin{array}{l}\text { Demanda espontânea, outras cidades ou associação } \\
\text { beneficente }\end{array}$ & 21 & 34,3 & 40 & 65,6 & \\
\hline \multicolumn{6}{|l|}{ Uso de drogas $(n=154)$} \\
\hline Drogas lícitas & 22 & 33,3 & 44 & 66,7 & \multirow{3}{*}{0,81} \\
\hline Não tem vício & 19 & 31,7 & 41 & 68,3 & \\
\hline Drogas ilícitas & 7 & 25,0 & 21 & 75,0 & \\
\hline
\end{tabular}

Fonte: Sagrilo KK, et al., 2020.

Verificou-se que a maioria dos participantes possuía valor de CD4+ menor que 200 células, portanto, houve associação entre uso de TARV e valor de $\operatorname{CD} 4+(p=0,001)$. A associação entre uso de TARV e CV foi estatisticamente significante $(p<0,0001)$, sendo frequente o não uso de TARV em pacientes com CV maior que 10.000 cópias. Também houve associação entre uso de TARV e tempo de diagnóstico $(p=0,002)$, sendo frequente o não uso de TARV em pacientes com menos de um ano de diagnóstico (Tabela 2). 
Tabela 2 - Análise descritiva das características clínicas das 156 pessoas vivendo com HIV (PVHIV), conforme o uso de TARV e valores de probabilidade $(p)$ associados ao teste de Qui-quadrado, 2017.

\begin{tabular}{|c|c|c|c|c|c|}
\hline \multirow{3}{*}{ Variáveis clínicas } & \multicolumn{4}{|c|}{ Uso de TARV } & \multirow{3}{*}{ Valor de $p$} \\
\hline & \multicolumn{2}{|c|}{$\operatorname{Sim}_{(n=49)}$} & \multicolumn{2}{|c|}{$\begin{array}{c}\text { Não } \\
(n=107)\end{array}$} & \\
\hline & $\mathrm{n}$ & $\%$ & $n$ & $\%$ & \\
\hline \multicolumn{6}{|c|}{ LT-CD4+ em 2015 (células/mm3) (n=154) } \\
\hline$>500$ & 14 & 53,8 & 12 & 46,2 & \multirow{3}{*}{0,001} \\
\hline 201 a 499 & 18 & 43,9 & 23 & 56,1 & \\
\hline$<200$ & 17 & 19,5 & 70 & 80,5 & \\
\hline \multicolumn{6}{|c|}{ Carga viral em 2015 (cópias $/ \mathrm{ml}$ ) (n=153) } \\
\hline Não detectável até $<40$ & 32 & 78,0 & 9 & 22,0 & \multirow{3}{*}{$<0,0001$} \\
\hline 40 a10.000 & 9 & 33,3 & 18 & 66,7 & \\
\hline$>10.000$ & 8 & 9,4 & 77 & 90,6 & \\
\hline \multicolumn{6}{|c|}{ Tempo de diagnóstico do HIV (anos) ( $\mathrm{n}=156)$} \\
\hline$<1$ & 3 & 8,1 & 34 & 91,9 & \multirow{5}{*}{0,002} \\
\hline 1 a 5 & 16 & 47,1 & 18 & 52,9 & \\
\hline 6 a 10 & 8 & 23,5 & 26 & 76,5 & \\
\hline 11 a 15 & 10 & 37,0 & 17 & 63,0 & \\
\hline 16 a 30 & 12 & 50,3 & 12 & 50,0 & \\
\hline \multicolumn{6}{|c|}{$\begin{array}{l}\text { Número de internações após o diagnóstico de } \\
\text { HIV ( } n=156)\end{array}$} \\
\hline Uma & 17 & 29,3 & 41 & 70,7 & \multirow{3}{*}{0,90} \\
\hline Duas a quatro & 20 & 33,3 & 40 & 66,7 & \\
\hline Cinco ou mais & 12 & 31,6 & 26 & 68,4 & \\
\hline
\end{tabular}

Fonte: Sagrilo KK, et al., 2020.

Dentre os principais motivos de internação, destacam-se: tuberculose, toxoplasmose cerebral, monilíase, pneumonia, diarreia, pneumocistose, hepatites, sífilis e herpes (Tabela 3).

Tabela 3 - Principais motivos de internação das 156 pessoas vivendo com HIV (PVHIV), 2017.

\begin{tabular}{|c|c|c|c|c|}
\hline $\begin{array}{c}\text { Internação de } 2015 \\
(n=156)\end{array}$ & $\begin{array}{c}\text { Primeira } \\
\text { internação }(n=98)\end{array}$ & $\begin{array}{c}\text { Segunda } \\
\text { internação } \\
(n=65)\end{array}$ & $\begin{array}{l}\text { Terceira } \\
\text { internação } \\
(n=49)\end{array}$ & $\begin{array}{l}\text { Quarta internação } \\
\qquad(\mathrm{n}=38)\end{array}$ \\
\hline $\begin{array}{c}\text { Tuberculose } \\
(n=32 ; 20,5 \%)\end{array}$ & $\begin{array}{c}\text { Tuberculose }(n=13 ; \\
13,3 \%)\end{array}$ & $\begin{array}{c}\text { Toxoplasmose } \\
\text { cerebral } \\
(\mathrm{n}=11,16,9 \%)\end{array}$ & $\begin{array}{c}\text { Toxoplasmose } \\
\text { cerebral } \\
(\mathrm{n}=12,24,5 \%)\end{array}$ & $\begin{array}{c}\text { Toxoplasmose } \\
\text { cerebral } \\
(\mathrm{n}=11 ; 28,9 \%)\end{array}$ \\
\hline $\begin{array}{c}\text { Monilíase } \\
(\mathrm{n}=32 ; 20,5 \%)\end{array}$ & $\begin{array}{c}\text { Toxoplasmose } \\
\text { cerebral } \\
(n=13 ; 13,3 \%)\end{array}$ & $\begin{array}{c}\text { Pneumonia }(n=8 \\
12,3 \%)\end{array}$ & $\begin{array}{c}\text { Pneumonia }(n=8 ; \\
16,3 \%)\end{array}$ & $\begin{array}{c}\text { Monilíase } \\
(n=6 ; 15,8 \%)\end{array}$ \\
\hline $\begin{array}{c}\text { Toxoplasmose } \\
\text { cerebral } \\
(n=29 ; 18,6 \%)\end{array}$ & $\begin{array}{c}\text { Pneumonia }(n=10 \\
10,2 \%)\end{array}$ & $\begin{array}{c}\text { Tuberculose }(\mathrm{n}=6 ; \\
9,2 \%)\end{array}$ & $\begin{array}{c}\text { Diarreia } \\
(\mathrm{n}=6 ; 12,2 \%)\end{array}$ & $\begin{array}{c}\text { Pneumonia } \\
(\mathrm{n}=4 ; 10,5 \%)\end{array}$ \\
\hline $\begin{array}{l}\text { Pneumocistose } \\
(n=25 ; 16,0 \%)\end{array}$ & $\begin{array}{l}\text { Causas obstétricas } \\
\quad(n=10 ; 10,2 \%)\end{array}$ & $\begin{array}{c}\text { Diarreia } \\
(n=5,7,7 \%)\end{array}$ & $\begin{array}{c}\text { Monilíase } \\
(n=5 ; 10,2 \%)\end{array}$ & $\begin{array}{c}\text { Hepatite ou } \\
\text { hepatopatias } \\
(n=4 ; 10,5 \%)\end{array}$ \\
\hline $\begin{array}{c}\text { Sífilis } \\
(n=19 ; 12,2 \%)\end{array}$ & $\begin{array}{c}\text { Candidíase }(\mathrm{n}=10 \\
10,2 \%)\end{array}$ & $\begin{array}{l}\text { Monilíase } \\
(\mathrm{n}=4 ; 6,2 \%)\end{array}$ & $\begin{array}{c}\text { Infecção do trato } \\
\text { urinário } \\
(n=5 ; 10,2 \%)\end{array}$ & $\begin{array}{l}\text { Tuberculose } \\
(n=3 ; 7,9 \%)\end{array}$ \\
\hline $\begin{array}{c}\text { Herpes } \\
(n=18 ; 11,5 \%)\end{array}$ & $\begin{array}{c}\text { Pneumocistose } \\
(\mathrm{n}=7 ; 7,1 \%)\end{array}$ & $\begin{array}{l}\text { Herpes zoster } \\
(\mathrm{n}=4 ; 6,2 \%)\end{array}$ & $\begin{array}{c}\text { Diabetes } \\
(n=3 ; 6,1 \%)\end{array}$ & $\begin{array}{c}\text { Diarreia } \\
(n=3 ; 7,9 \%)\end{array}$ \\
\hline $\begin{array}{l}\text { Pneumonia } \\
(\mathrm{n}=16 ; 10,3 \%)\end{array}$ & $\begin{array}{c}\text { Diarreia } \\
(n=6 ; 6,1 \%)\end{array}$ & $\begin{array}{c}\text { Infecção do trato } \\
\text { urinário } \\
(n=3 ; 4,6 \%)\end{array}$ & $\begin{array}{l}\text { Intoxicação } \\
\text { exógena } \\
(n=3 ; 6,1 \%)\end{array}$ & $\begin{array}{l}\text { Neurocriptococose } \\
\quad(n=2 ; 1,3 \%)\end{array}$ \\
\hline $\begin{array}{c}\text { Hepatite ou } \\
\text { hepatopatias ( } \mathrm{n}=16 ; \\
10,3 \%)\end{array}$ & $\begin{array}{l}\text { Hepatite ou } \\
\text { hepatopatia } \\
(n=5 ; 5,1 \%)\end{array}$ & $\begin{array}{c}\text { Esofagite ou } \\
\text { gastrite } \\
(n=3 ; 4,6 \%)\end{array}$ & $\begin{array}{c}\text { Crise convulsiva } \\
(n=3 ; 6,1 \%)\end{array}$ & $\begin{array}{l}\text { Os outros motivos } \\
\text { ocorreram uma única } \\
\text { vez }(n=1 ; 0,6 \%)\end{array}$ \\
\hline
\end{tabular}

Fonte: Sagrilo KK, et al., 2020. 


\section{DISCUSSÃO}

$\mathrm{Na}$ amostra estudada houve predomínio do sexo masculino, indivíduos com 40 anos ou mais e baixa escolaridade. A maior frequência de PVHIV em homens é compatível com o perfil da epidemia do HIV/Aids no Brasil. Atualmente, a doença atinge 26 homens para cada 10 mulheres (BRASIL, 2019). Entretanto, um estudo destacou a necessidade de implementar estratégias educativas nos serviços de saúde para o cuidado à mulher vivendo com HIV, considerando-se aspectos singulares como transmissão vertical (LINDER V, et al., 2016).

Em estudo realizado por Chaves LL, et al. (2020), a prevalência do HIV/Aids acentuada em homens foi explicada devido ao comportamento de risco que essa população possui, pelo fato de grande parte não utilizar o preservativo e procurar o serviço de saúde tardiamente para o diagnóstico. Além disso, quando se relaciona o gênero à adesão ao tratamento, Souza HC, et al. (2018) verificou que possuem menor adesão ao tratamento e mais episódios de prognósticos negativos.

O nível de escolaridade menor esteve frequente na maioria dos participantes que não tinham adesão a TARV. Em um estudo que verificou a adesão a TARV por meio de um questionário de adesão ao tratamento, observou-se que os participantes com maior nível de escolaridade apresentavam adesão adequada. Isso se deve a melhor compreensão dos participantes com mais anos de estudo sobre a patologia e a terapêutica medicamentosa, refletindo na adesão (SOUZA HC, et al., 2018).

Neste estudo, a maior parte não fazia uso de TARV, o que pode estar relacionado ao motivo da internação, em consequência da grave imunodeficiência que a não adesão ao tratamento causa. Este cenário é um desafio para o setor público de saúde. Portanto, deve-se avaliar os dilemas de adesão de cada indivíduo, considerando: as doses dos medicamentos, eventos adversos, rede de atenção à saúde, disposição e vontade do paciente e a rede das relações das PVHIV (ROSSI PS e PEREIRA PPG, 2014; COSTA JO, et al., 2020).

Em estudo realizado na Austrália foram evidenciados diversos fatores que afetam a adesão ao tratamento de PVHIV/Aids, dentre eles: idade avançada e fatores relacionados ao medicamento como efeitos colaterais e número de doses, que acarreta em uma adesão não adequada (COSTA JO, et al., 2020). Em uma revisão integrativa também foram identificados múltiplos fatores associados à adesão à TARV incluindo características individuais, aspectos relacionados ao tratamento e com a infecção, a relação com o serviço de saúde e o apoio social (CARVALHO PP, et al., 2019).

Em estudo realizado no Ceará, em que se verificou a adesão a TARV em coinfectados HIV e Tuberculose, observou-se que $86,5 \%$ dos participantes apresentavam adesão inadequada ao tratamento. A adesão foi avaliada por autorrelato do paciente, utilizando-se o instrumento Questionário para Avaliação da Adesão ao Tratamento Antirretroviral.

Para aqueles com HIV/TB, o cumprimento do esquema terapêutico é difícil em razão de terem que usar medicamentos concomitantes para tratar as duas infecções. Essa situação pode ter contribuído para que a adesão à TARV, neste estudo, fosse predominantemente inadequada (LEMOS LA, et al., 2016).

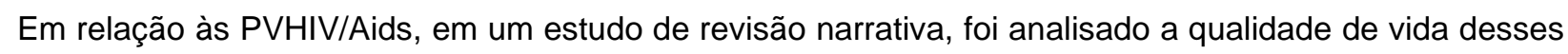
indivíduos, considerada de extrema importância no que diz respeito à compreender o modo com que a pessoa convive com esse agravo tão estigmatizante e como isso influencia ao seu plano terapêutico (BELTRAO PPL, et al., 2020).

Dentre as variáveis estudadas, a faixa etária foi associada ao não uso de TARV, com menor uso entre os indivíduos mais jovens. Resultados similares foram encontrados em estudo desenvolvido na Bahia, em que pacientes com menos de 34 anos apresentaram 2,2 vezes mais chances de não aderir à TARV, comparados com aqueles com 34 ou mais (SILVA JAG, et al., 2015).

A associação entre faixa etária e adesão também foi significante em estudo realizado no interior paulista, com maior frequência de adesão adequada ou estrita em indivíduos com 40 anos ou mais (FORESTE JS, et al., 2017). 
Em estudo realizado em Pernambuco, corroborando a prerrogativa da feminização da doença e sua heterossexualização bem como em relação a TARV, $2 \%$ das mulheres em estudo não aderiram ao tratamento e 4,5\% o abandonaram (TORRES SSBM, et al., 2020).

Os participantes do presente estudo apresentaram contagem baixa de células T CD4+ e CV elevada, parâmetros clínicos insatisfatório do controle do HIV, o que propicia a instalação da Aids. Estudos demonstram que grande parte das mortes de PVHIV que ocorrem no período de internação hospitalar estão relacionadas às Infecções Oportunistas (IOs) (COELHO LE, et al., 2016). Esses achados reforçam a importância dos serviços de saúde adotarem medidas para fortalecer o diagnóstico e tratamento precoce a fim de minimizar, em longo prazo, a incidência de IOs e internações hospitalares (BARRENECHE MFA, et al., 2017; SAAVEDRA A, et al., 2017).

No presente estudo, o valor de CD4+ e a CV foram associadas ao não uso de TARV, sendo mais frequente o não uso de TARV em pacientes com CD4+ menor que 200 células $/ \mathrm{mm} 3$ e com CV maior que 10.000 cópias. Resultados similares foram encontrados em um estudo realizado no interior paulista em que se observou maior percentual de adesão adequada ou estrita no grupo de indivíduos com CD4+ maior que 350 (FORESTE JS, et al., 2017). Esses resultados eram esperados, considerando-se que o exame de CV contribui na identificação de indivíduos que tem problemas com adesão. Ressalta-se ainda que a TARV é a principal intervenção comprovadamente eficaz na redução da morbimortalidade das PVHIV (BRASIL, 2018).

$\mathrm{Na}$ amostra estudada, o não uso de TARV foi frequente em pacientes com menos de um ano de diagnóstico. Corroborando com este resultado, um estudo desenvolvido em dois Serviços de Atendimento Especializado a PVHIV, a adesão adequada ou estrita foi mais frequente em indivíduos com cinco anos ou mais de diagnóstico (FORESTE JS, et al., 2017). Esses achados sugerem a necessidade de fortalecimento do vínculo de PVHIV com a equipe e serviço de saúde durante o período de adaptação à TARV, com enfoque na avaliação e no manejo de eventos adversos tardios e dificuldades relacionadas à adesão em longo prazo (BRASIL, 2018).

As IOs, definidoras do quadro de Aids, foram as principais causas de hospitalização durante o período, com destaque para tuberculose, toxoplasmose cerebral, monilíase e pneumonia. Em um estudo realizado em um município do Pará, observou-se que as IOs com maior prevalência também foram a tuberculose, seguida da neurotoxoplasmose e candidíase oral (CHAVES LL, et tal., 2020). Esses achados sugerem que as IOs ainda são responsáveis pelo aumento das internações hospitalares entre as PVHIV mesmo no período pós TARV (OLIVEIRA RB, et al., 2014).

O paciente ao fazer o uso adequado dos medicamentos, na dose e posologia indicadas, obtém melhorias que resultam da supressão viral, como a redução dos episódios de IOs e consequente hospitalização, aumentando a qualidade de vida e diminuindo os índices de mortalidade (SILVA RTS, et al., 2016).

Pesquisas apontam que o acesso aos serviços de saúde, para diagnóstico e tratamento, a promoção da autonomia do sujeito como responsável pelo seu próprio autocuidado, as relações com os profissionais e o acompanhamento com permanente avaliação pela equipe de saúde são fatores essenciais para a promoção da adesão à terapia medicamentosa (SILVA RTS, et al., 2016; SILVA ACO, et al., 2014).

A associação entre o uso de TARV e número de internações hospitalares após o diagnóstico de HIV não obteve significância estatística, mas esse resultado diverge de outras pesquisas que relatam que o início tardio e o uso inadequado dos antirretrovirais resultam em um elevado número de pacientes com quadro de imunossupressão ou de IOs, achado que explica a alta ocorrência de internações hospitalares (NUNES AA, et al., 2015; SILVA JAG, et al., 2015).

É relevante salientar os dados ignorados quanto as variáveis demográficas, trazendo uma reflexão do quanto é importante adquiri-los durante a notificação. Deve-se obter o maior número de informações possíveis visto que esses dados serão fonte de informações para o planejamento de ações por parte do serviço, promovendo políticas públicas para o enfrentamento dessa epidemia, visto que esses dados funcionam também como um importante indicador da vulnerabilidade tanto da população em geral quanto das PVHIV. 
O presente estudo apresentou resultados relevantes sobre o uso de TARV, destacam-se: a elevada frequência de não uso desta terapia, a elevada frequência de não uso em indivíduos jovens e em pacientes com tempo de diagnóstico menor de um ano. Dessa forma, ressaltamos a necessidade de melhoria na implantação de políticas voltadas à adesão à TARV, incluindo, encontros periódicos de PVHIV com toda equipe de saúde para avaliar os motivos de não adesão e orientar quanto aos riscos relacionados a não adesão.

Destaca-se a necessidade de atividades específicas voltadas ao indivíduo jovem, incluindo a investigação dos motivos e/ou obstáculos que se antepõe aos pacientes, retardando sua chegada aos serviços de saúde.

Muitos pacientes com HIV desconhecem que possuem a infecção e, dessa forma, não buscam o serviço de saúde até que os sinais da Aids se manifestem. Isso vem de encontro com os principais motivos de internação, sendo estes, as IOs.

Tais achados reforçam a importância de propor promoção de ações nas áreas de prevenção, além do uso de preservativos, disponibilizar acesso às profilaxias pós e pré exposição (PEP e PrEP) e disponibilizar o autoteste em unidade de saúde. Ressalta-se, ainda, a necessidade de assegurar ao indivíduo o acesso oportuno à unidade de saúde após exposições. Devem ser realizadas atividades que encorajem as pessoas a se testarem com o intuito de descobrirem o quadro precocemente e iniciarem o tratamento.

\section{CONCLUSÃO}

O contexto analisado retrata o não uso de TARV por PVHIV associado a indivíduos jovens, tempo de diagnóstico menor de um ano, com internações por IOs que poderiam ser evitadas. A existência de fatores desfavoráveis ao uso inadequado de TARV representa um grande desafio para a saúde pública. Assim, conhecendo as causas mais frequentes de dificuldade de adesão ao tratamento, é imprescindível a abordagem por meio de medidas educativas, tanto para os usuários, quanto aos profissionais de saúde. Devese promover mudanças no acolhimento, vínculo, humanização, ações de prevenção, acompanhamento presente, em médio e longo prazo, e ainda a oferta de uma rede de apoio engajada entre a atenção primária à saúde, centro de referência e as unidades de internação. Nesse sentido vale enfatizar a importância da melhoria do acesso e da acessibilidade a assistência à saúde, o qual apresenta potencialidades para favorecer a práxis, sobremaneira no incentivo à adesão, diminuindo, assim, as fragilidades na sua operacionalização.

\section{REFERÊNCIAS}

1. BARRENECHE MFA, et al. Hospitalization causes and outcomes in HIV patients in the late antiretroviral era in Colombia. AIDS Research and Therapy, 2017; 14:60.

2. BELTRÃO PPL, et al. Saúde e qualidade de vida das pessoas vivendo com HIV/aids: uma revisão narrativa dos últimos 15 anos. Revista Eletrônica Acervo em Saúde, 2020; 40:e2942.

3. BRASIL MS. Secretaria de Vigilância em Saúde. Departamento de Doenças de Condições Crônicas e Infecções Sexualmente Transmissíveis - DCCI. Boletim epidemiológico HIV/AIDS, 2019:1-70.

4. BRASIL MS. Secretaria de Vigilância em Saúde. Departamento de Vigilância, Prevenção e Controle das Infecções Sexualmente Transmissíveis, do HIV/Aids e das Hepatites Virais. Protocolo Clínico e Diretrizes Terapêuticas para Manejo da Infecção pelo HIV em Adultos, 2018:1-412.

5. CARVALHO PP, et al. Fatores associados à adesão à terapia antirretroviral em adultos: revisão integrativa de literatura. Ciências \& Saúde Coletiva, 2019; 24(7):2543-55.

6. CHAVES LL, et al. Prevalência de infecções oportunistas em pacientes HIV positivos atendidos no Centro de Testagem e Aconselhamento (CTA) em município do Pará, em 2015 e 2016. Revista Eletrônica Acervo Saúde, 2020; 51:1-9.

7. COELHO LE, et al. Predictors of opportunistic illnesses incidence in post combination antiretroviral therapy era in an urban cohort from Rio de Janeiro, Brazil. BMC Infectious Diseases, 2018; 16:134.

8. COSTA JO, et al. Adherence to antiretroviral regimens in Australia: a nationwide cohort study. AIDS Patient Care STDS, 2020; 34(2):81-91

9. FORESTE JS, et al. Adherence to antiretroviral therapy by people living with HIV/AIDS in a municipality of São Paulo. Revista Gaúcha Enfermagem, 2017; 38(1):e63158.

10. LEMOS LA, et al. Adherence to antiretrovirals in people coinfected with the human immunodeficiency virus and tuberculosis. Revista Latino-Americana Enfermagem, 2016; 24:e2691. 
11. LINDER V, et al. Percepções de mulheres vivendo com o vírus da imunodeficiência humana acerca da impossibilidade de amamentar. Enfermagem em Foco, 2016; 7(2):07-11.

12. MALTA M, et al. Iniciativa STROBE: subsídios para a comunicação de estudos observacionais. Revista Saúde Pública, 2010; 44(3):559-65.

13. MORAES TM, et al. Percepções de trabalhadores técnico-administrativos de um curso de enfermagem do estado do Pará sobre HIV/Aids. Enfermagem em foco, 2019; 10 (4): 17-20.

14. NUNES AA, et al. Análise do perfil de pacientes com HIV/Aids hospitalizados após introdução da terapia antirretroviral (HAART). Ciências Saúde Coletiva, 2015; 20(10):3191-98.

15. OLIVEIRA RB, et al. Epidemiology of invasive fungal infections in patients with acquired immunodeficiency syndrome a at reference hospital for infectious diseases in Brazil. Mycopathologia, 2014; 178(1-2):71-8.

16. RAVANHOL GM, et al. Pessoas vivendo com HIV/Aids no cárcere: regularidade no uso da terapia antirretroviral. Acta Paulista de Enfermagem, 2019;32(5):521-9.

17. ROSSI PS, PEREIRA PPG. O remédio é o menor dos problemas: seguindo redes na adesão ao tratamento de Aids. Saúde e Sociedade, 2014; 23(2):484-95

18. SAAVEDRA A, et al. Causes of death and factors associated with early mortality of HIV-infected adults admitted to Korle-Bu Teaching Hospital. The Pan African Medical Journal, 2017; 27:48

19. SILVA ACO, et al. Quality of life, clinical characteristics and treatment adherence of people living with HIV/AIDS. Revista Latino-Americana Enfermagem, 2014; 22(6):994-1000.

20. SILVA HFN, et al. Avaliação do tratamento antirretroviral de pessoas convivendo com HIV/Aids que participam de um grupo de adesão. Medicina (Ribeiro Preto Online), 2019; 52(3):161-70.

21. SILVA JAG, et al. Factors associated with non-adherence to antiretroviral therapy in adults with AIDS in the first six months of treatment in Salvador, Bahia State, Brazil. Caderno Saúde Pública, 2015; 31(6):1188-98

22. SILVA RTS, et al. Perfil clínico-epidemiológico de adultos hiv-positivo atendidos em um hospital de Natal/RN. Revista de Pesquisa: Cuidado é Fundamental, 2016; 8(3):4689-96.

23. SOUZA HC, et al. Análise da adesão ao tratamento com antirretrovirais em pacientes com HIV/AIDS. Revista Brasileira de Enfermagem, 2019; 72(5):1361-9.

24. TORRES SSBM, et al. Perfil da mulher portadora de HIV/AIDS e sua adesão ao tratamento. Revista Eletrônica Acervo em Saúde, 2020; 12(10):e4041.

25. UNAIDS. Global AIDS update. 2019.

26. UNAIDS. Global AIDS update. 90-90-90 Uma meta ambiciosa de tratamento para contribuir para o fim da de Aids. 2015. 